Pacific

Journal of

Mathematics

EFFECTIVE DIVISOR CLASSES ON A RULED SURFACE

JEFF ROSOFF

Volume $202 \quad$ No. 1

January 2002 


\title{
EFFECTIVE DIVISOR CLASSES ON A RULED SURFACE
}

\author{
JEFF RosofF
}

The Neron-Severi group of divisor classes modulo algebraic equivalence on a smooth algebraic surface is often not difficult to calculate, and has classically been studied as one of the fundamental invariants of the surface. A more difficult problem is the determination of those divisor classes which can be represented by effective divisors; these divisor classes form a monoid contained in the Neron-Severi group. Despite the finite generation of the whole Neron-Severi group, the monoid of effective divisor classes may or may not be finitely generated, and the methods used to explicitly calculate this monoid seem to vary widely as one proceeds from one type of surface to another in the standard classification scheme (see Rosoff, 1980, 1981).

In this paper we shall use concrete vector bundle techniques to describe the monoid of effective divisor classes modulo algebraic equivalence on a complex ruled surface over a given base curve. We will find that, over a base curve of genus 0 , the monoid of effective divisor classes is very simple, having two generators (which is perhaps to be expected), while for a ruled surface over a curve of genus 1 , the monoid is more complicated, having either two or three generators. Over a base curve of genus 2 or greater, we will give necessary and sufficient conditions for a ruled surface to have its monoid of effective divisor classes finitely generated; these conditions point to the existence of many ruled surfaces over curves of higher genus for which finite generation fails.

\section{Preliminaries on ruled surfaces.}

Let $C$ be a nonsingular complex curve, and let $X$ be a ruled surface over $C$. Then ([H1, V.2.2, p. 370]) $X$ can be written as $\mathbf{P}(E)$, the projectivization of a rank 2 vector bundle $E$ on $C$. Moreover, for rank 2 bundles $E$ and $E^{\prime}, \mathbf{P}(E)=\mathbf{P}\left(E^{\prime}\right)$ if and only if $E$ and $E^{\prime}$ differ by a twisting with a line bundle.

Let $X$ be a ruled surface over the base curve $C$, say $X=\mathbf{P}\left(E^{\prime}\right)$. Replacing $E^{\prime}$ by $E=E^{\prime} \otimes L$ for some line bundle $L$ chosen appropriately, we may assume that $E$ is normalized so that: 
(a) $E$ admits a nontrivial global section, and,

(b) $E \otimes L$ admits no nontrivial section for any line bundle $L$ of negative degree.

$E$ is determined up to twisting with line bundles of degree 0 . The choice of a (normalized) bundle $E$ to represent $X$ as the projectivization of a rank two bundle determines the linear equivalence class of a "standard" section $C_{0}$ of $X$ over $C$, with $\mathcal{O}_{X}\left(C_{0}\right)=\mathcal{O}_{\mathbf{P}(E)}(1)$.

If $E$ is a rank 2 bundle on $C$, then $\operatorname{deg} \Lambda^{2}(E)=\operatorname{deg} \Lambda^{2}(E \otimes L)$ for any line bundle $L$ of degree 0 , and hence is a well-defined invariant of $X=\mathbf{P}(E)$ for $E$ normalized as above. Finally, the Neron-Severi group of $X$ is a free group of rank 2, generated by the algebraic equivalence classes of the section $C_{0}$ and of any fiber $F$ of the natural map $\pi: X \rightarrow C$. Denoting by $[D]$ the algebraic equivalence class of any divisor $D$, the intersection pairing on $X$ is given by:

$$
\left[C_{0}\right]^{2}=\operatorname{deg} \Lambda^{2}(E), \quad\left[C_{0}\right] \bullet[F]=1, \quad[F]^{2}=0 .
$$

1. Ruled surfaces with $\left[C_{0}\right]^{2} \leq 0$.

Theorem 1. Let $C$ be a smooth curve and let $X=\mathbf{P}(E)$ be a ruled surface over $C$ such that, with $E$ normalized as above, $\left[C_{0}\right]^{2}=\operatorname{deg} \Lambda^{2}(E) \leq 0$. Then the monoid of effective divisor classes on $X$ is $\left\{a\left[C_{0}\right]+b[F] \mid a, b \geq 0\right\}$.

Remark. Since any vector bundle over $\mathbf{P}^{1}$ splits into a direct sum of line bundles, this theorem applies to any ruled surface over a curve of genus 0 ; a normalized rank 2 bundle will be of the form $\mathcal{O} \oplus L$ for some line bundle $L$ of degree $\leq 0$. These are the "Hirzebruch surfaces".

Proof. Clearly any divisor class in the above set represents an effective divisor. Now, let $D$ be an effective divisor on $X$, so that $D$ can be written as $D=n C_{0}+\sum D_{i}$ with $n \geq 0$ and $D_{i}$ an irreducible curve other than $C_{0}$. Letting $\left[D_{i}\right]=a_{i}\left[C_{0}\right]+b_{i}[F]$, we have that $\left[D_{i}\right] \bullet[F]=a_{i} \geq 0$ and $\left[D_{i}\right] \bullet\left[C_{0}\right]=b_{i}+a_{i}\left[C_{0}\right]^{2} \geq 0$, so $b_{i} \geq 0$. The result follows by additivity.

\section{Ruled surfaces over curves of genus 1 .}

Theorem 2. Let $X=\mathbf{P}(E)$ be a ruled surface over an elliptic curve $C$, with $E$ normalized. Then the monoid of effective divisor classes on $X$ is (finitely) generated by:

(a) $\left[C_{0}\right]$ and $[F]$ if $\operatorname{deg} \Lambda^{2}(E) \leq 0$, and

(b) $\left[C_{0}\right],[F]$ and the anti-cannonical class $2\left[C_{0}\right]-[F]$ if $\operatorname{deg} \Lambda^{2}(E)>0$.

Proof. We may assume that $\operatorname{deg} \Lambda^{2}(E) \geq 1$, and by Nagata [N, pp. 191-96] we may assume that this degree is 1 .

Since $E$ has a nontrivial section and since $E \otimes \mathcal{O}(-p)$ has none for any $p \in C$, we have an exact sequence of bundles $0 \rightarrow \mathcal{O}_{C} \rightarrow E \rightarrow \mathcal{O}(p) \rightarrow 0$, 
with $E$ a nontrivial extension of $\mathcal{O}(p)$ by $\mathcal{O}_{C}$ for some fixed $p \in C$. The nontriviality of the extension corresponds to $E$ being normalized.

We will first show that the divisor class $2\left[C_{0}\right]-[F]$ contains an effective divisor, i.e., that the bundle $\mathcal{O}_{X}(2) \otimes \pi^{*} \mathcal{O}_{C}(-q)$ has nontrivial section for some $q \in C$. By $\left[\mathbf{H 1}, 7.11\right.$, p. 162] $\pi_{*} \mathcal{O}_{X}(2)=S^{2}(E)$, the second symmetric power of $E$, so by the projection formula it suffices to show that $S^{2}(E) \otimes$ $\mathcal{O}_{C}(-q)$ has a section for some $q \in C$.

The structure of the space of indecomposable bundles of a given rank and degree on an elliptic curve has been extensively studied by Atiyah $[\mathbf{A}$, pp. 414-52], and we will appeal to his results to show that $S^{2}(E)=\sum \mathcal{O}\left(p_{i}\right)$ where the $\left\{p_{i}\right\}$ are the three nontrivial half-periods of $C$ under the translation sending $p$ to the origin. In Atiyah's notation $E=e_{A}(2,1)$ where $A$ is the bundle $\mathcal{O}(p)$, and by [A, Th. 7, p. 434], $\check{E}=e_{A}(2,-1)=E \otimes \mathcal{O}(-p)$ [A, Th. 6, p. 433].

Thus, $E \otimes E \otimes \mathcal{O}(-p)=E \otimes \check{E}=$ End $(E)=\mathcal{O}_{C} \oplus \sum \mathcal{O}\left(p_{i}-p\right)$ with the $\left\{p_{i}\right\}$ as above using [A, Lemma 22, p. 439], and so $E \otimes E=\mathcal{O}(p) \oplus \sum \mathcal{O}\left(p_{i}\right)$. On the other hand, over $\mathbf{C}, E \otimes E=S^{2}(E) \oplus \Lambda^{2}(E)=S^{2}(E) \oplus \mathcal{O}(p)$. Thus $S^{2}(E)=\sum \mathcal{O}\left(p_{i}\right)$ and $S^{2}(E) \otimes \mathcal{O}(-q)$ has a section for $q \in\left\{p_{i}\right\}$.

Thus the monoid generated by the classes $\left[C_{0}\right],[F]$ and $2\left[C_{0}\right]-[F]$ is contained in the monoid of effective divisor classes on $X$. The reverse inclusion follows directly from [H1, V.2.21, p. 382].

\section{Ruled surfaces over curves of higher genus.}

For the reminder of this article, we will assume that our ruled surface $X$ is the projectivization of a normalized rank 2 bundle $E$ on the curve $C$ with $g(C) \geq 1$ and, in view of Theorem 1 , that $\operatorname{deg} \Lambda^{2}(E) \geq 1$.

By $[\mathbf{H 1}, \mathrm{V} .2 .21$, p. 382], if $Y$ is an irreducible curve on $X$ with $[Y]=$ $a\left[C_{0}\right]+b[F]$, then Hurwitz's theorem applied to the desingularization of $Y$ shows that either $a, b \geq 0$, or $a \geq 2$ and $b \geq-(a / 2) \operatorname{deg} \Lambda^{2}(E)$. It follows from linearity that these are necessary conditions for an algebraic equivalence class to contain an effective divisor.

On the other hand, sufficient conditions are provided by the RiemannRoch theorem: By Serre duality $\operatorname{dim} H^{2}\left(X, \mathcal{O}_{X}\left(a C_{0}+b F\right)\right)=0$ for $a \geq 0$, and Riemann-Roch on the surface $X$ applied to the divisor class $\left[a C_{0}+b F\right]$ shows that this class contains an effective divisor if $a \geq 0$ and $b \geq g-$ $(a / 2) \operatorname{deg} \Lambda^{2}(E)$.

Theorem 3. Let $X=\mathbf{P}(E)$ be a ruled surface over a curve $C$ with $g(C) \geq$ 1 , and $\operatorname{deg} \Lambda^{2}(E) \geq 1$ with $E$ normalized. Then the monoid of effective divisor classes on $X$ is finitely generated if and only if there is effective divisor class $a\left[C_{0}\right]+b[F]$ with $a \geq 2$ and $b=-(a / 2) \operatorname{deg} \Lambda^{2}(E)$. 
Proof. Suppose that there is an effective divisor class as above, and let $A \geq 2$ be minimal such that the class $A\left[C_{0}\right]-(A / 2) \operatorname{deg} \Lambda^{2}(E)[F]$ contains an effective divisor, say $D_{0}$. Let $S=\left\{a\left[C_{0}\right]+b[F] \mid 1 \leq a<A\right.$ and $\left.-(a / 2) \operatorname{deg} \Lambda^{2}(E) \leq b<0\right\} ; S$ contains a finite number of (not necessarily effective) divisor classes, say $\left[E_{1}\right] \cdots\left[E_{k}\right]$.

Let $\breve{S}=\left\{\left[E_{i}\right] \in S \mid\left[E_{i}\right]+n\left[D_{0}\right]\right.$ contains an effective divisor for some $n \geq 0\}$, and for each $\left[E_{i}\right] \in \check{S}$ let $D_{i}=E_{i}+n_{i} D_{0}$ with $n_{i} \geq 0$ minimal in the above regard. Then the monoid of effective divisor classes on $X$ is (finitely) generated by the classes $\left[C_{0}\right],[F]$ and the $\left[D_{i}\right], i \geq 0$.

Conversely, suppose that there is no effective divisor class on $X$ meeting the condition of the theorem. Then, for any finite collection of (nontrivial) effective divisors $D_{1}, \ldots, D_{k}$ we have $\left[D_{i}\right]=a_{i}\left[C_{0}\right]+b_{i}[F]$ with $a_{i} \geq 0$ and $b_{i}>-\left(a_{i} / 2\right) \operatorname{deg} \Lambda^{2}(E)$, i.e., for some $\varepsilon_{i}>0 b_{i} \geq-\left(a_{i} / 2\right) \operatorname{deg} \Lambda^{2}(E)+a_{i} \varepsilon_{i}$. For any nonnegative integers $c_{1}, \ldots, c_{k}$ (not all 0 ) the linear combination $A\left[C_{0}\right]+B[F]=c_{1}\left[D_{1}\right]+\cdots+c_{k}\left[D_{k}\right]$ has $B \geq-(A / 2) \operatorname{deg} \Lambda^{2}(E)+A \varepsilon$ for $\varepsilon=\min \left\{\varepsilon_{i}\right\}, \varepsilon>0$.

Finally, select an integer a sufficiently large so that $g(C)<\varepsilon a$; making $a$ even if necessary, let $b \in \mathbf{Z}$ be $b=g(C)-(a / 2) \operatorname{deg} \Lambda^{2}(E)$. By our above observation on Riemann-Roch, the divisor class $a\left[C_{0}\right]+b[F]$ is an effective class, but cannot be expressed as a nonnegative linear combination of the classes $\left\{\left[D_{i}\right]\right\}$. Thus, if there is no effective class as in the theorem, the monoid of effective divisor classes on $X$ is not finitely generated.

A geometric criterion for finite generation of the monoid of effective classes is given by:

Corollary. The monoid of effective divisor classes on $X$ is finitely generated if and only if there is a curve $Y$ on $X$ such that the projection $\pi: X \rightarrow C$ exhibits $Y$ as an unramified $n$-fold cover of $C$ for some $n \geq 2$.

Proof. Indeed, the proof of [H1, V.2.21, p. 382] shows that an irreducible curve $Y$ on $X$ that is an unramified $n$-fold cover of $C$ (for $n \geq 2$ ) must necessarily have $[Y]=n\left[C_{0}\right]-(n / 2) \operatorname{deg} \Lambda^{2}(E)[F]$, and that any curve in a divisor class of this form (with $n$ minimal and $n \geq 2$ ) is such a cover.

Remark. For any such curve $Y$ on $X$ as above, we must have $\operatorname{dim} H^{0}(X$, $\left.\mathcal{O}_{X}(Y)\right)=1$. To see this, note that if $D \in|Y|$, then $D \cap \pi^{-1}(u)$ consists of $n$ distinct points for all $u \in C$. If there are two linearly independent sections $s_{i}$ in $H^{0}\left(X, \mathcal{O}_{X}(Y)\right)$, then as $Y^{2}=0$, we have a surjection $\Psi: X \rightarrow \mathbf{P}^{1}$ given by $\Psi(p)=\left[s_{1}(p), s_{2}(p)\right]$. Since $\Psi^{-1}(t) \in|Y|$ for $t \in \mathbf{P}^{1}, \Psi \mid F$ gives an unramified $n$-fold cover of $\mathbf{P}^{1}$ for a fiber $F$, which is impossible for $n>1$.

Theorem 4. Let $X$ be the ruled surface $\mathbf{P}(E)$ over the curve $C$ with $g(C)$ $\geq 1$, with $E$ normalized and with $\operatorname{deg} \Lambda^{2}(E)>0$. Then finite generation of the monoid of effective divisor classes on $X$ is equivalent to the existence 
of a sub-line bundle $N$ of $S^{n}(E)$ with $\operatorname{deg}(N)=(n / 2) \operatorname{deg} \Lambda^{2}(E)$, for some $n>1$.

Proof. $[\mathbf{H 2}, 10.2$, p. 51] shows that a multisection $Y$ of $\mathbf{P}(E)$ of degree $n$ over $C$ corresponds to a sub-line bundle $N$ of $S^{n}(E)$, and further that the requirement that $[Y]=n\left[C_{0}\right]-(n / 2) \operatorname{deg} \Lambda^{2}(E)[F]$ forces $\operatorname{deg}(N)=$ $(n / 2) \operatorname{deg} \Lambda^{2}(E)$.

Remark. An interesting geometric proof of Theorem 3 can be obtained by considering the projective $n$-bundle $\mathbf{P}\left(S^{n} \check{E}\right)$ over $C$, with projection $\psi$ to $C$. Giving a multisection $Y$ of $\mathbf{P}(E)$ of degree $n$ over $C$ can be viewed as giving a section $\sigma$ of $\mathbf{P}\left(S^{n} \check{E}\right)$ - this is really the same as giving local homogeneous equations defining $Y$ on each fiber of $\pi$ in $\mathbf{P}(E)$, with the fibers of $\psi$ parameterizing homogeneous polynomials of degree $n$ in two variables. There is a divisor $\Delta$ on $\mathbf{P}\left(S^{n} \check{E}\right)$ whose intersection with each fiber of $\psi$ corresponds to such local equations having zero discriminant; a linear algebra computation shows that $O(\Delta)=\mathcal{O}(2 n-2) \otimes \psi^{*}(L)$ for some line bundle $L$ on $C$ of degree $n(n-1) \operatorname{deg} \Lambda^{2}(E)$. The requirement that $Y$ be an unramified $n$-fold cover of $C$ is that $\sigma(C)$ not meet $\Delta$, so that $\operatorname{deg} \sigma^{*}(\Delta)=0$, i.e., $\operatorname{deg} \sigma^{*} \mathcal{O}(1)=-(n / 2) \operatorname{deg} \Lambda^{2}(E)$ using $n \geq 2$. Since $\sigma^{*} \mathcal{O}(1)$ is a quotient of $S^{n}(\check{E})[\mathbf{H 1}, 7.12$, p. 162], we may take for the bundle $N$ above the dual $\sigma^{*} \mathcal{O}(-1)$.

Remark. For the ruled surface $\mathbf{P}(E)$ over an elliptic curve $C$ with $\operatorname{deg} \Lambda^{2}(E)$ $=1$, there are precisely three sub-line bundles of $S^{2}(E)$ of the correct degree: The $\mathcal{O}\left(p_{i}\right)$ in the proof of Theorem 2 .

Remark. It is known [H2, 10.5, pp. 53-54] that, on a curve $C$ of genus $g(C) \geq 2$, there are bundles of any given degree and rank that are stable and all of whose symmetric powers are also stable. For such a rank 2 bundle $E, E \otimes L$ has the same property for any line bundle $L$. Thus, even with $E$ normalized if necessary we will have, for any sub-line bundle $N$ of $S^{n}(E)$, $\operatorname{deg}(N)<(n / 2) \operatorname{deg} \Lambda^{2}(E)$. For such a bundle $E$ whose normalizations have positive degree, the monoid of effective divisor classes on the ruled surface $\mathbf{P}(E)$ requires an infinite number of generators.

\section{References}

[A] M.F. Atiyah, Vector bundles over an elliptic curve, Oxford University Press, Proceedings London Math. Soc., 7(3) (1957), 414-452, MR 24 \#A1274, Zbl 084.17305.

[H1] R. Hartshorne, Algebraic Geometry, Graduate Text in Mathematics, 52, SpringerVerlag, 1977, MR 57 \#3116, Zbl 367.14001.

[H2] _ Ample Subvarieties of Algebraic Varieties, Lectures Notes in Math., 156, Springer-Verlag, 1970, MR 44 \#211, Zbl 208.48901. 
[N] M. Nagata, On self-intersection number of a section on a ruled surface, Nagoya Math., 37 (1970), 191-196, MR 41 \#3475 Zbl 193.21603.

[R1] J. Rosoff, Effective divisor classes and blowings-up of $\mathbf{P}^{2}$, Pacific J. Math., 89(2) (1980), 419-429, MR 82e:14042, Zbl 564.14002.

[R2] - The monoid of effective divisor classes on a complex torus, Springer-Verlag, Lectures Notes in Math., 862 (1981), 208-231, MR 83m:14032, Zbl 488.14002.

Received August 12, 1999 and revised August 7, 2000. The author would like to thank the mathematics departments at the New Zeland Universities of Canterbury and Otago for their hospitality during the preparation of this manuscript, and to the referee for many valuable suggestions.

Gustavus Adolphus College

St. Peter, MN 56082

E-mail address: jr@gac.edu 